\title{
Reference values for spirometry in Chinese aged 4-80 years
}

\author{
Wenhua Jian $^{1 *}$, Yi Gao ${ }^{1 *}$, Chuangli $\mathrm{Hao}^{2}$, Ning Wang ${ }^{3}$, Tao $\mathrm{Ai}^{4}$, Chuanhe $\mathrm{Liu}^{5}$, Yongjian $\mathrm{Xu}^{6}$, Jian $\mathrm{Kang}^{7}$, \\ Lan Yang ${ }^{8}$, Huahao Shen', Weijie Guan ${ }^{1}$, Mei Jiang ${ }^{1}$, Nanshan Zhong ${ }^{1}$, Jinping Zheng ${ }^{1}$
}

${ }^{1}$ State Key Laboratory of Respiratory Disease, National Clinical Research Center for Respiratory Disease, Guangzhou Institute of Respiratory Diseases, First Affiliated Hospital of Guangzhou Medical University, Guangzhou 510120, China; ${ }^{2}$ Soochow University Affiliated Children's Hospital, Soochow 215025, China; ${ }^{3}$ Xi'an Children's Hospital, Xi'an 710003, China; ${ }^{4}$ Chengdu Women and Children's Central Hospital, Chengdu 610017, China; ${ }^{5}$ The Capital Institute of Pediatrics, Beijing 100045, China; ${ }^{6}$ Tongji Hospital, Tongji Medical College, Huazhong University of Science \& Technology, Wuhan 430074, China; ${ }^{7}$ The First Hospital of China Medical University, Shenyang 110001, China; ${ }^{8}$ The First Affiliated Hospital of Xi'an Jiaotong University, Xi'an 710061, China; ${ }^{9}$ The Second Affiliated Hospital of Zhejiang University School of Medicine, Hangzhou 310009, China

Contributions: (I) Conception and design: All authors; (II) Administrative support: All authors; (III) Provision of study materials or patients: All authors; (IV) Collection and assembly of data: All authors; (V) Data analysis and interpretation: All authors; (VI) Manuscript writing: All authors; (VII) Final approval of manuscript: All authors.

*These authors contributed equally to this work.

Correspondence to: Jinping Zheng. State Key Lab of Respiratory Disease, National Clinical Research Center for Respiratory Disease, Guangzhou Institute of Respiratory Diseases, First Affiliated Hospital of Guangzhou Medical University, No.151 Yanjiang Road, Guangzhou 510120, China. Email: jpzhenggy@163.com.

Background: Although there are over 1.34 billion Chinese in the world, nationwide spirometric reference values for Chinese are unavailable, which is usually based on Caucasian conversion. The aim of this study was to establish spirometric reference values for Chinese with a national wide sample.

Methods: We enrolled healthy non-smokers in 24 centers in Northeast, North, Northwest, Southwest, South, East and Central China from January 2007 to June 2010. Spirometry was performed according to American Thoracic Society and European Respiratory Society guidelines. Reference equations were established using the Lambda-Mu-Sigma (LMS) method for forced expiratory volume in 1 second $\left(\mathrm{FEV}_{1}\right)$, forced vital capacity (FVC), $\mathrm{FEV}_{1} / \mathrm{FVC}$, peak expiratory flow (PEF) and maximal midexpiratory flow (MMEF). Popular Caucasian reference values adjusted with ethnic conversion factors were validated with Chinese measured spirometry data. The present study also compared with other published Chinese equations for spirometry.

Results: A total of 7,115 eligible individuals aged 4 to 80 years (50.9\% females) were recruited. Reference equations against age and height by gender were established, including predicted values and lower limits of normal (LLNs). Validated with Chinese data, the mean percentage differences of Caucasian reference values adjusted with ethnic conversion factors were $-10.2 \%$ to $1.8 \%$, and the percentages of total subjects under LLNs were $0.1 \%$ to $8.9 \%$. Compared with this study, the percentage differences of previous Chinese studies ranged from $-17.8 \%$ to $11.4 \%$, which were found to significantly overestimate or underestimate lung function.

Conclusions: This study established new reference values for better interpretation of spirometry in Chinese aged 4 to 80 years, while Caucasian references with adjustment were inappropriate for Chinese.

Keywords: Lung function; spirometry; predicted values; lower limits of normal (LLNs); Lambda-Mu-Sigma (LMS) method; Chinese

Submitted Sep 02, 2017. Accepted for publication Oct 17, 2017.

doi: $10.21037 /$ jtd.2017.10.110

View this article at: http://dx.doi.org/10.21037/jtd.2017.10.110 


\section{Introduction}

Spirometry has been widely used for diagnosing respiratory diseases, quantifying disease severity, and assessing disease prognosis $(1,2)$. Accurate interpretation of spirometry requires appropriate reference values derived from its own ancestry population (3), including lower limits of normal (LLNs), which could be helpful for assessment of abnormal pulmonary function in patients with pulmonary diseases.

There are over 40 million overseas Chinese (4) and 1.3 billion mainland Chinese (5) in the world (about 22\% of the global population), indicating the huge medical demand (6). Embarrassingly, standardized nationwide spirometric reference values for Chinese were unavailable.

In 2012, Global Lung Function Initiative (3) recommended multi-ethnic reference values for African-Americans, Southeast Asians (SEA-GLI2012) and Northeast Asians (NEA-GLI2012), which were largely established with Caucasian data and adjusted with fixed ethnic conversion factors in the whole age range. In addition, other Caucasian reference values adjusted with fixed ethnic conversion factors were also applied in China $(7,8)$, such as European Committee of Steel and Coal equations adjusted for Chinese with the suggestion of Zheng et al. (Chinese-ECSC1993) $(9,10)$, and the third national health and nutrition examination surveys equations adjusted with 0.88 times for Asian-American (Asian-NHANESIII 0.88) (11,12). Given the dynamic changes of gene, economic, environment, nutrition and et al., it remains unknown whether those fixed ethnic conversion factors reliably reflect the difference of spirometry between Caucasians and Chinese.

Although several spirometric reference values for Chinese have been published (13-22), the major disadvantages in these studies limited the nationwide use, including small samples, limited age ranges, small local regions, as well as different study protocols and quality control. Without LLNs for nationwide Chinese, a fixed 0.7 of forced expiratory volume in 1 second to forced vital capacity $\left(\mathrm{FEV}_{1} / \mathrm{FVC}\right)$ instead of LLNs was frequently applied for the diagnosis of "airflow limitation" in previous studies $(7,23,24)$, leading possible underdiagnosis in younger subjects and over diagnosis in elderly. Moreover, In the nationwide questionnaire surveys on clinical application of pulmonary function testing conducted in 2002 (25) and 2012 (unpublished data), the majority $(78.6 \%$ and $77.8 \%$, respectively) of hospitals in China were unaware of where the reference values were derived from and used the default reference values in spirometers only. Even in the same region, different hospitals used various references. These could lead to improper assessment of pulmonary function and treatment effects.

Consequently, it is essential to establish ethnic reference values for spirometry in Chinese with a large sample size and wide age range, and validate whether fixed ethnic conversion factors reflect the differences between Caucasian and Chinese in the whole age range.

\section{Methods}

\section{Study design}

This cross-sectional study was conducted with the uniform study protocol, between January 2007 and December 2010, at 24 collaborative centers across China. Approval was obtained from local ethics committee. Written informed consent was obtained from participants or their guardians (when participants were under 18 years of age). All centers selected are in the region that lived with a big amount of population, among them, majority are Han people, and minority groups (non-Han) were excluded in the present study.

\section{Subjects}

Adult participants were recruited in health screening department of each center, while children participants were recruited in kindergartens, elementary and middle schools that closed to centers, in which all children in the same class were selected. The occupation of participants included preschool children, students, teachers, farmers, workers, office staffs, retirees, etc. The key inclusion criteria were: (I) life-long non-smokers; (II) no symptoms and history of chronic cardiopulmonary diseases (chronic bronchitis, asthma, lung cancer, pulmonary fibrosis, pulmonary tuberculosis, chronic heart diseases, etc.); (III) no abnormal findings on physical examination; (IV) written informed consent was obtained. The key exclusion criteria were: (I) upper or lower respiratory infection within 4 weeks; (II) long-term exposure to harmful gas or particles; (III) using $\beta$-blocker for treatment; (IV) pregnant women, epileptic; (V) other diseases or surgeries potentially affecting lung function. Health questionnaires about medical history and personal characters were provided to the participants and children's parents prior to spirometry. Age was calculated with the date of examination and the definite birthday. Standing height was recorded to the nearest $0.5 \mathrm{~cm}$ without shoes. Weight was measured to the nearest $0.5 \mathrm{~kg}$ with 
light-weight clothing.

\section{Spirometry}

Spirometers all met the instrument standardization of American Thoracic Society and European Respiratory Society (ATS-ERS) (26). Technicians were full-time staffs for spirometry in centers, and well-trained to follow the procedures and criteria recommended by ATS-ERS statements $(26,27)$. Prior to spirometry, calibration check was undertaken daily with a $3-\mathrm{L}$ syringe, and confirming that the variations were within limits, e.g., $\pm 3 \%$ (90 mL). With correction of sitting postures, subjects attached the nose clip or manual occlusion of the nares, placed mouthpiece in the mouth and closed their lips around the mouthpiece. Then they were encouraged to inhale completely and rapidly with a pause of $<1$ second at total lung capacity, and exhaled maximally until no more air could be expelled while maintaining an upright posture. Acceptable maneuvers included: (I) free from artefacts (cough during the first second of exhalation; glottis closure that influenced on the measurement; early termination or cutoff; effort that was not maximal; gas leakage or obstructed mouthpiece); (II) good starts (extrapolated volume less than $5 \%$ of $\mathrm{FVC}$ or $0.15 \mathrm{~L}$, whichever is greater); (III) satisfactory exhalation (a plateau in the volume-time curve or forced expiratory time of $\geq 3$ seconds in children aged $<10$ years, $\geq 6$ seconds in subjects aged $>10$ years or if the subject cannot or should not continue to exhale). Manoeuvers were repeated for 3 to 8 times in each subject until three acceptable and repeatable spirograms were obtained. For both FVC and $\mathrm{FEV}_{1}$, the acceptable difference was within $150 \mathrm{~mL}$ between the largest and second largest values. The largest FVC, $\mathrm{FEV}_{1}$ and peak expiratory flow (PEF) were selected within the best three maneuvers, while maximal midexpiratory flow (MMEF) were taken from the maneuver with the largest sum of FVC and $\mathrm{FEV}_{1}$. Spirometry was performed between 8:00 am and 12:00 am.

\section{Data collection}

Health questionnaires and original spirometry reports of participants after preliminary screening and physical examination were collected in each center and sent to the First Affiliated Hospital of Guangzhou Medical University for centering quality control, which was conducted by two well-trained physicians in accordance with ATS-ERS criteria $(26,27)$. If they had inconsistent conclusions with the same report, consultancy with a third senior physician would make the final decision. All data were double input and checked with Epidata 3.0 (Odense, Denmark). Data that yielded $\mathrm{z}$-scores $<-5.0$ or $>5.0$ were identified as outliers and excluded (3).

\section{Statistical analysis}

Spirometric reference equations for the whole subjects were derived using Lambda-Mu-Sigma (LMS) method $(28,29)$ using Generalized Additive Models of Location Scale package (GAMLSS version 4.3-1) in the statistical software $\mathrm{R}$ (version 3.1.2; http://www.r-project.org). The spirometry data were modeled with age and standing height as explanatory variables. Using the Box-Cox-Cole-Green distribution, the best model was estimated by exploring whether log transformations of dependent and independent variables were required. Considering of unnecessary over-fitting, the optimal degrees of freedom for the cubic spline curve were chosen by selecting the model with the smallest Schwarz Bayesian Criterion. In addition, the goodness of fit was judged from inspection of normal Q-Q plots, worm plots, the distribution of residuals as a function of age and predicted value, and from density plots of residuals. In keeping with Cole et al., log transformation of all the indices provided the best fit to the data. Thus, the reference equations were established as a function of age and height in males and females, including skewness (location, $L$ ), mean $(M)$ and coefficient of variation (scatter, S). $M$ indicates the predicted value as follows: $M=\exp [\mathrm{a}+\mathrm{b} \times \ln$ (height in $\mathrm{cm})+\mathrm{c} \times$ $\ln$ (age in year) $+M$-spline], where $a, b$, and $c$ are coefficients, and $\mathrm{M}$-spline is an age-specific contribution from the spline function. Likewise, LLN was calculated as follows: LLN (5th percentage $)=\exp [\ln (M)+\ln (1-1.645 \times L \times S) / L]$. Z-scores were calculated as (observed-predicted)/SD, where SD was calculated as (predicted-LLN)/1.645.

Similarly, a best fit of standing height for age was derived from the data of males and females with GAMLSS. In the validation analysis, the predicted values of this study and NEAGLI2012, SEA-GLI2012, Chinese-ECSC1993, and AsianNHANESIII 0.88 were compared with the measured data of spirometry; if the average z-score that yielded \pm 0.4 (30) or the percentage difference that yielded $\pm 5.0 \%$, the equations would not be considered as appropriate for Chinese. In addition, the new reference equations of this study were compared with other previous published reference equations derived from Chinese using assumptive anthropometric characters. 


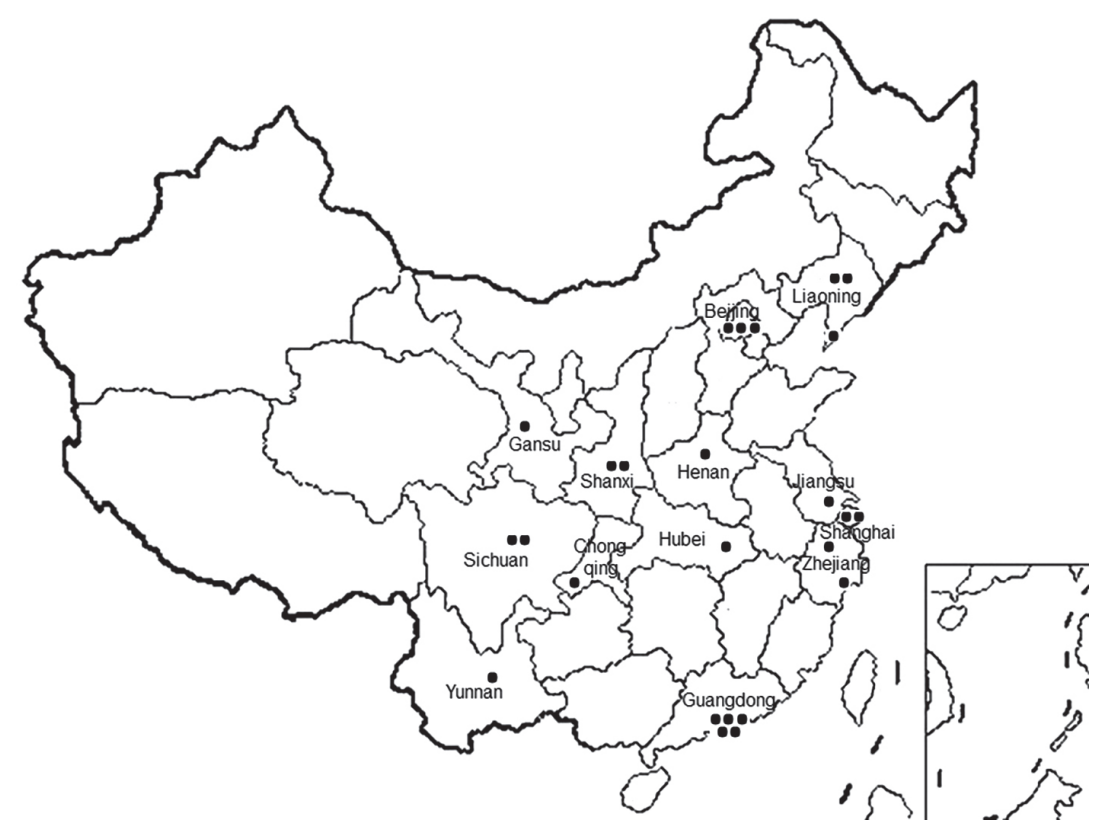

Figure 1 The 24 collaborative centers in China.

\section{Results}

\section{Subjects}

Figure 1 illustrates 24 collaborative centers in China. After preliminary screening of questionnaire and physical examination in each center, spirometry was performed with a total of 7,715 individuals initially. The numbers of exclusion and reasons are listed in Figure 2. No data yielded a z-score of $<-5.0$ or $>5.0$. Quality assurance data of 7,115 individuals (50.9\% females; 4 to 80 years old and 95.0 to $190.0 \mathrm{~cm}$ in height) was collected. The general geographic characterization is shown in Table 1. The distributions of age for males and females are demonstrated in Figure 3, where the intervals of age are 2 years in children (until 14 years of age in China).

\section{Reference equations established for spirometry}

The reference equations and look-up tables for FVC, $\mathrm{FEV}_{1}, \mathrm{FEV}_{1} / \mathrm{FVC}, \mathrm{PEF}$ and $\mathrm{MMEF}$ are presented as files in supplementary material (Tables S1-S3). There were nearly linear relationships between age, height and spirometric indices in adult data and children data taken separately, such as the measured $\mathrm{FEV}_{1}$ in Figure 4. The best fitting models required $\log$ transformations of spirometric indices, height and age in the whole data. The age-specific predicted values and LLNs are shown in Figure 5. The LLNs of $\mathrm{FEV}_{1} / \mathrm{FVC}$ were less than 0.70 in males above 56 years of age and in females above 60 years of age.

Validation of the popular Caucasian equations adjusted with fixed ethnic conversion factors

In Table 2, SEA-GLI2012 underestimated the FVC and $\mathrm{FEV}_{1}$ of the present study with the mean z-score ranged from 0.64 to $0.85(-10.0 \%$ to $-7.2 \%)$, while those measured values below LLNs $(\%<\mathrm{LLN})$ were $0.1 \%$ to $1.0 \%$. Although the mean percentage differences of FVC and $\mathrm{FEV}_{1}$ between NEA-GLI2012 and the present study were $-2.4 \%$ to $1.2 \%$, the z-scores against age were beyond +0.4 in children, especially in male children (Figure 6). Across the whole age in Figure 7, SEA-GLI2012 and NEAGLI2012 overestimated the \% < LLN of $\mathrm{FEV}_{1} / \mathrm{FVC}$ at most age range. In addition, Chinese-ECSC1993 and Asian-NHANESIII 0.88 also underestimated FVC and $\mathrm{FEV}_{1}$ of Chinese $(-8.8 \%$ to $-3.9 \%$, and $-10.2 \%$ to $-7.3 \%$, respectively), while those $\%<\mathrm{LLN}$ were $0.2 \%$ to $1.4 \%$ and $0.1 \%$ to $0.9 \%$, respectively.

\section{Comparisons with previous reference values derived from Chinese}

For $\mathrm{FEV}_{1}$ and FVC of adults aged 20, 40, and 60 years in Table 3, the percentage differences between this study and previous studies were $-2.0 \%$ to $12.9 \%$ in $\mathrm{Wu}$ et al. (20), 


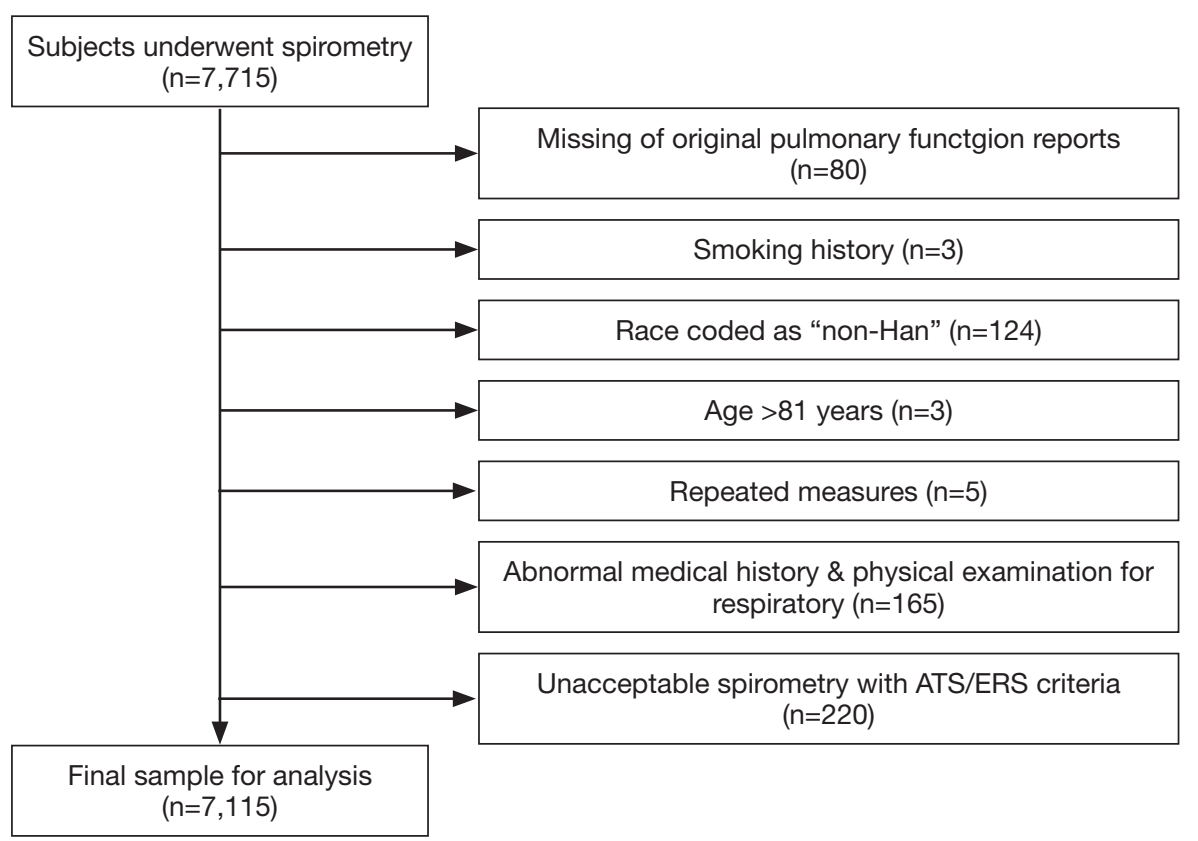

Figure 2 Flowchart of subjects' exclusion after quality check. ATS, American Thoracic Society; ERS, European Respiratory Society.

Table 1 The general geographic characterization of the present study

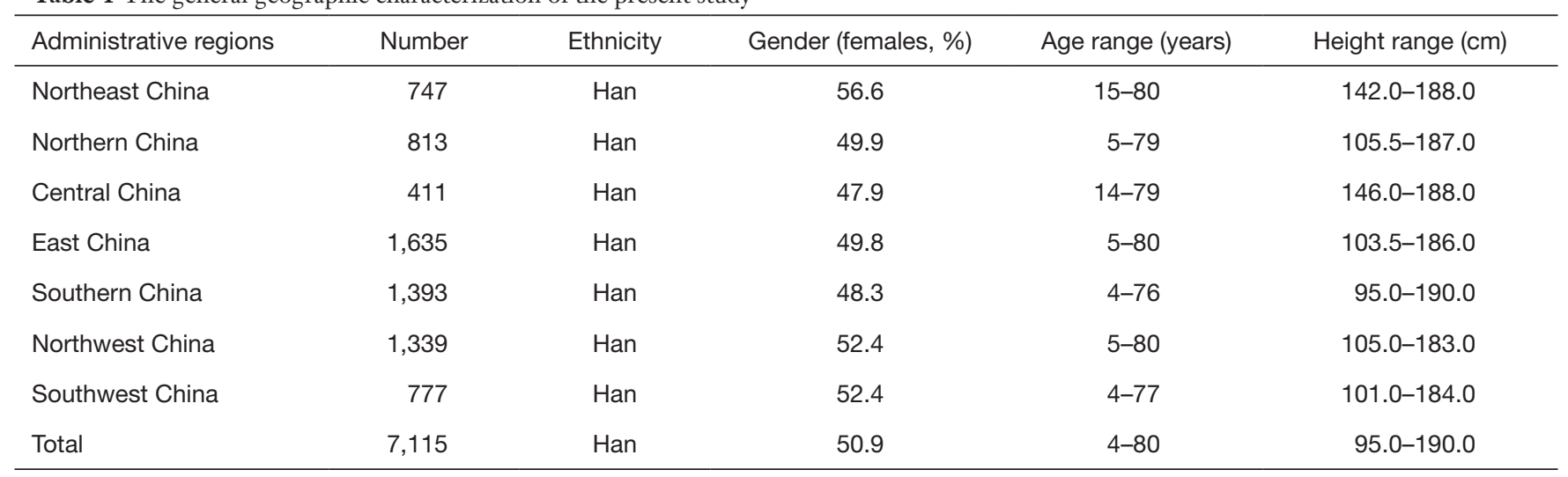

$-13.2 \%$ to $-4.6 \%$ in Ip et al. (16), $-9.2 \%$ to $3.1 \%$ in Pan et al. (17), respectively. For FEV $\mathrm{FV}_{1}$ and FVC of children aged 6, 10, and 14 years in Table 4, the percentage differences were $-17.8 \%$ to $-12.1 \%$ in Ma et al. $(21),-7.0$ to $-0.9 \%$ in Ip et al. (15), respectively.

\section{Discussion}

Predicted values and LLNs for spirometry in Chinese aged 4 to 80 years were established with 7,115 non-smoking health subjects in 24 collaborative centers. The present study demonstrated that Caucasian reference values adjusted with fixed ethnic conversion factors were inappropriate for Chinese in the whole age range. Compared with this study, the previous Chinese studies were found to significantly overestimate or underestimate lung function measurements for Chinese.

This study showed that SEA-GLI2012 and NEAGLI2012 significantly underestimated or overestimated the $\mathrm{FEV}_{1}, \mathrm{FVC}$, and $\mathrm{FEV}_{1} / \mathrm{FVC}$ for Chinese, not matter 
in percentage differences, $\mathrm{z}$-scores or $\%<\mathrm{LLNs}$. To our knowledge, SEA-GLI2012 and NEA-GLI2012 included Chinese, Koreans and Thais, while Chinese were from Hong Kong $(15,16)$, Taiwan (17), Shenzhen (13) and Northeast China (20) only, whom were insufficient to reflect the whole Chinese population. Furthermore, pooling data from those studies between 1990 and 2009 might cause the bias with different study protocols and secular trends for lung function. In addition, GLI2012 did not include PEF, an important index, which could help the management of asthma (2) and screening of chronic obstructive pulmonary disease (COPD) (31). The present study also demonstrated that Chinese-ECSC1993 and Asian-NHANESIII 0.88 underestimated Chinese in $\mathrm{FVC}$ and $\mathrm{FEV}_{1}$, while the adjustment of $\mathrm{FEV}_{1} / \mathrm{FVC}$, PEF and MMEF were not available in these equations. In the above reference

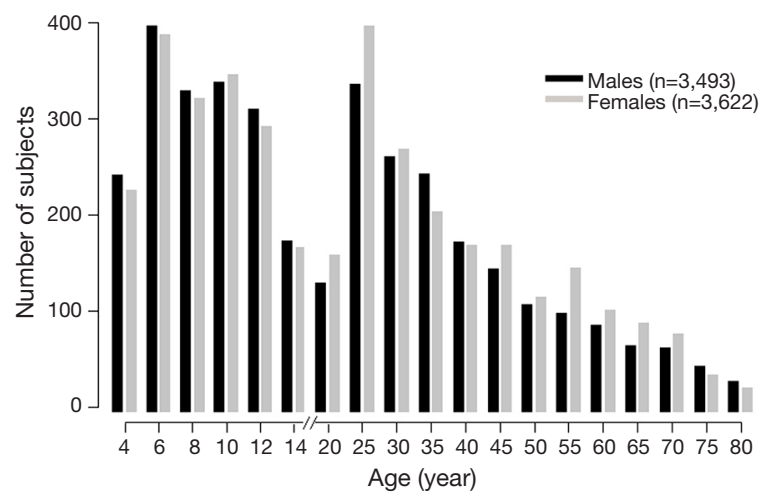

Figure 3 Age distribution in the final sample $(n=7,115)$. The intervals of age are 2 years in children (until 14 years of age in China) and 5 years in other age ranges.

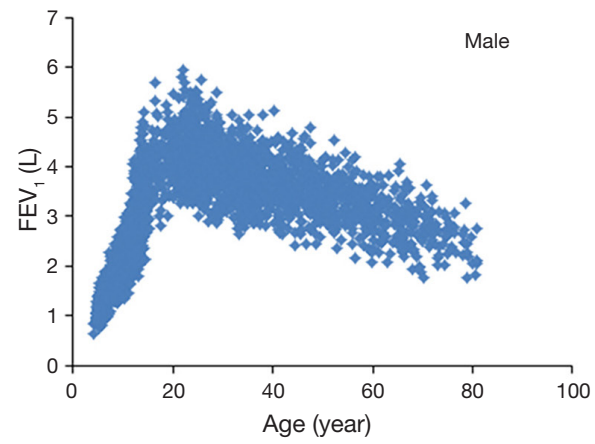

equations, as well as GLI2012, fixed correction factors were used to adjust ethnic differences, which were not be proper for all ages.

This study compared with the previous studies for Chinese. Ip et al. (16) and Pan et al. (17) significantly underestimated the FVC and $\mathrm{FEV}_{1}$ of adults (up to $-13.2 \%$ ), while $\mathrm{Wu}$ et al. (20) significantly overestimated the $\mathrm{FVC}$ and $\mathrm{FEV}_{1}$ of female adults (up to $11.4 \%$ ). In the other hand, Ma et al. (21) and Ip et al. (15) both underestimated the $\mathrm{FVC}$ and $\mathrm{FEV}_{1}$ of children (up to $-17.8 \%$ ). Moreover, the reference values of $\mathrm{FEV}_{1} / \mathrm{FVC}$ for children were rarely reported in the previous studies, and this issue should be paid attention in clinical practice. Anyway, the momentous changes of economy, life styles, nutritional status, and living environment have been observed in recent decades, which might impact significantly on pulmonary function $(16,32)$.

What we taken critical concern is the fact that there are no local contemporary reference values in most regions of Chinese, except for Caucasian reference values adjusted with ethnic conversion factors, while it could be impractical to establish reference for each region. With huge medical service demands (6), it is essential and urgent to establish a unique representing reference for Chinese, which is not only conducive for the government and Centers for Disease Control to make the policies, and take actions for the management and prevention of respiratory diseases, but also good for assessment of individuals. In addition, increasing domestic and international multi-center clinical trials have been conducted, in which unified reference equations for spirometry are compulsory.

Without LLNs of $\mathrm{FEV}_{1} / \mathrm{FVC}$, a fixed ratio 0.70 of $\mathrm{FEV}_{1} / \mathrm{FVC}$ was widely used in clinical practice and

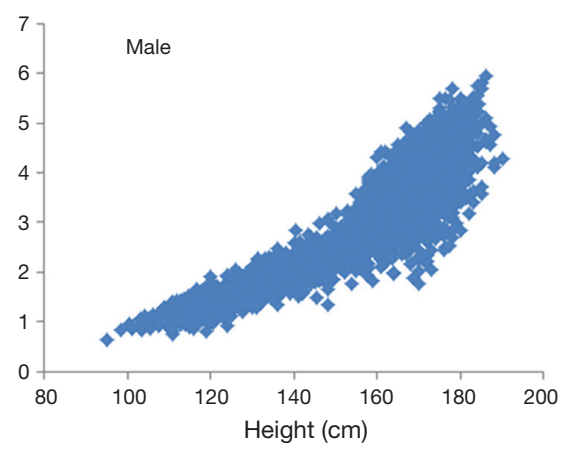

Figure 4 The measured $\mathrm{FEV}_{1}$ against age and height in males. $\mathrm{FEV}_{1}$, forced expiratory volume in 1 s; FVC, forced vital capacity; SEAGLI2012 and NEA-GLI2012, multi-ethnic Global Lung Initiative 2012 equations for Southeast and Northeast Asian; Chinese-ECSC1993, European Committee of Steel and Coal equations adjusted for Chinese; Asian-NHANESIII 0.88, the third National Health and Nutrition Examination Surveys equations adjusted with 0.88 times for Asian-American. 

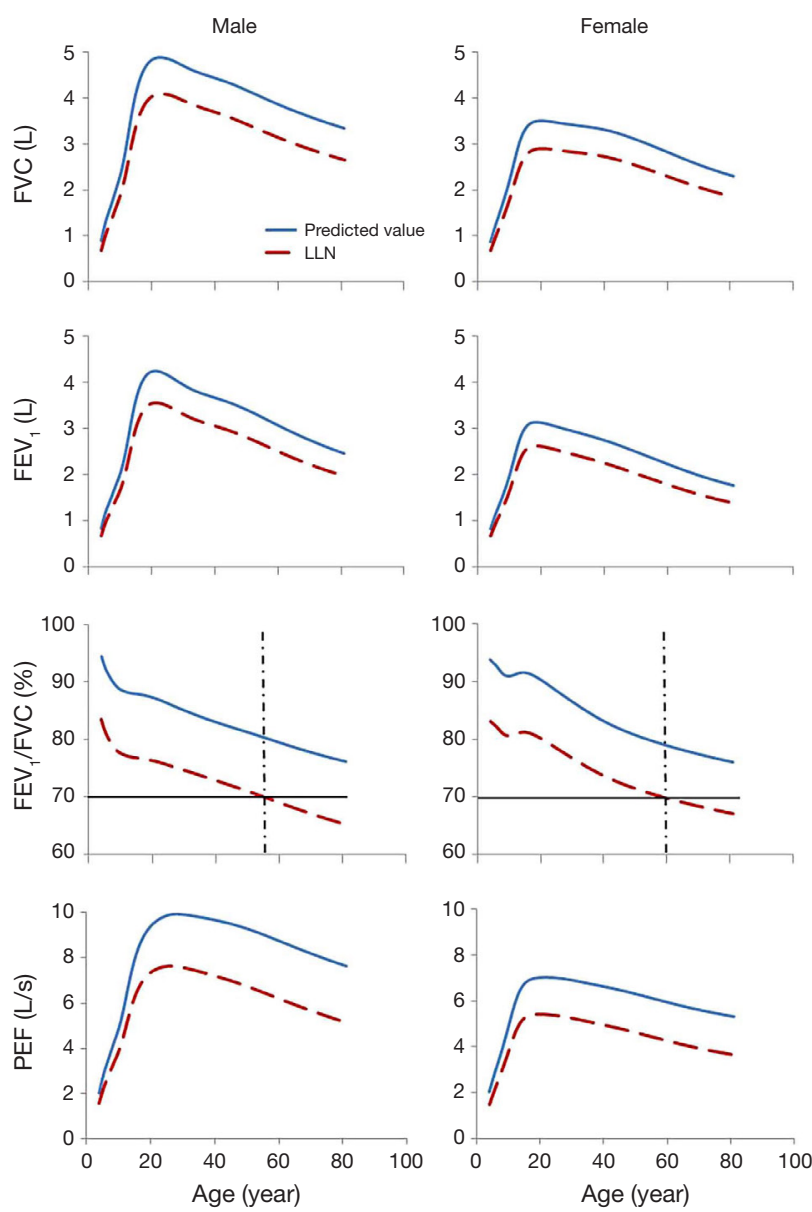

Figure 5 Predicted values and LLNs in males and females for FVC, $\mathrm{FEV}_{1}, \mathrm{FEV}_{1} / \mathrm{FVC} \%$, PEF, respectively. Graphs were generated using mean height for age in the present study. LLNs, lower limits of normal; $\mathrm{FEV}_{1}$, forced expiratory volume in 1 second; FVC, forced vital capacity; PEF, peak expiratory flow.

epidemiological research in China $(7,23)$, while this fixed $\mathrm{FEV}_{1} / \mathrm{FVC}$ particularly went against the studies of early COPD due to possible underdiagnosis in younger subjects and over diagnosis in elderly. Although our study protocol for intervention in Chinese patients with early COPD has been published (24), the accuracy of diagnosis for COPD with the fixed 0.7 of $\mathrm{FEV}_{1} / \mathrm{FVC}$ is still questioned. Instead, LLNs were suggested to provide more accurate diagnosis in Caucasians (33). The controversy that whether the LLN or the fixed 0.7 is better for clinical practice still needs further longitudinal study to clarify. Undoubtedly, the present study provides the LLNs of spirometric indices for Chinese, an important reference for clinical practice.

There are some limitations in the present study. We did not record the influences of pulmonary function, such as economic conditions, residential environment, nutrition, etc. Because of the frequent immigrant in the life, it is hard to clearly definite which one from the rural/urban area, not in our study either. The present study enrolled the Han people only, but Han accounts for $91.51 \%$ of the total population in China (5). Han can be considered as a representative of Chinese, owing to no available prediction equations of lung function for minority groups, as well as, the living environment and ancestor of non-Han that are close to those of Han. Considering of the difficulty for recruiting the healthy non-smokers aged $>80$ years, the subjects within such age range were excluded. However, the reference values for Chinese aged 4 to 80 years met the most demands of clinical practice. Because the healthy participants were enrolled from healthy screening department, we just recorded those who met the inclusion/ exclusion criteria and completed the spirometry. Therefore, these issues will be concerned in future studies.

\section{Conclusions}

In conclusion, the present study established new reference values including LLNs for better interpretation of spirometry in Chinese. Spirometric reference values between different races (such as Caucasian and Chinese) might not be adjusted with fixed ethnic conversion factors for all ages.

\section{Acknowledgements}

The following people and centers contributed data to this manuscript. Chen Wang, Beijing Chaoyang Hospital, Capital Medical University, Beijing 100020, China; Zhiyang Luo, Foshan First People's Hospital, Foshan 528000, China; Zhenshan Wang, The Second Hospital of Dalian Medical University, Dalian 116027, China; Ping Chen, The General Hospital Of Shenyang Military Region, Shenyang 110016, China; Wanzhen Yao, Peking University Third Hospital, Beijing 100191, China; Fuqiang Wen, West China Hospital of Sichuan University, Chengdu 610041, China; Zhengxian Chen, Guangdong General Hospital, Guangzhou 510080, China; Yuanrong Dai, The Second Affiliated Hospital \& Yuying Children's Hospital of Wenzhou Medical University, Wenzhou 325027, China; Changzheng Wang, Xinqiao Hospital, Third Military Medical University, Chongqing 400037, China; Jinming Liu, Shanghai Pulmonary Hospital, Shanghai 200433, China; Guihua Zhao, Henan Provincial People's Hospital, Zhengzhou 450003, China; Huanying 

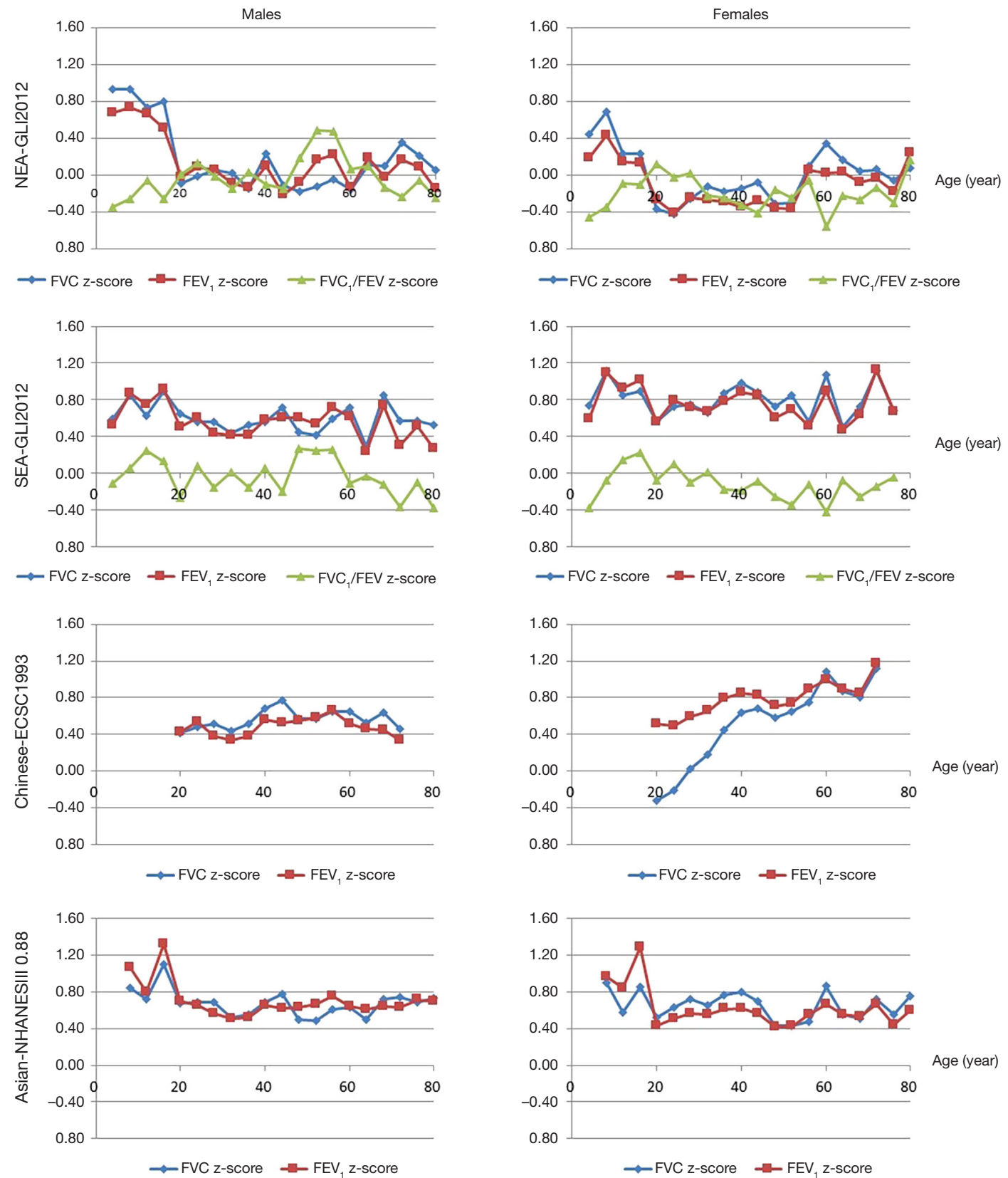

Figure 6 The z-score of the measured values across the whole age in forced vital capacity (FVC, blue line), forced expiratory volume in 1 second $\left(\mathrm{FEV}_{1}\right.$, red line) and $\mathrm{FEV}_{1} / \mathrm{FVC}$ (green line). The intervals of age are 5 years. SEA-GLI2012 and NEA-GLI2012, multi-ethnic Global Lung Initiative 2012 equations for Southeast and Northeast Asian; Chinese-ECSC1993, European Committee of Steel and Coal equations adjusted for Chinese; Asian-NHANESIII 0.88, the third National Health and Nutrition Examination Surveys equations adjusted with 0.88 times for Asian-American; FVC, forced vital capacity; $\mathrm{FEV}_{1}$, forced expiratory volume in 1 second. 


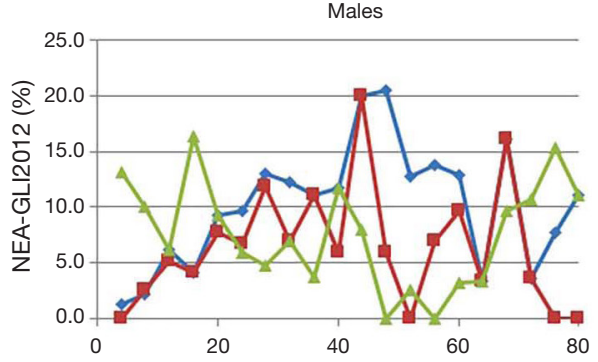

$\rightarrow$ FVC $\%<$ LLN-E-FEV $1 \%<$ LLN $\rightarrow$ FEV 1 /FVC $\%<$ LLN

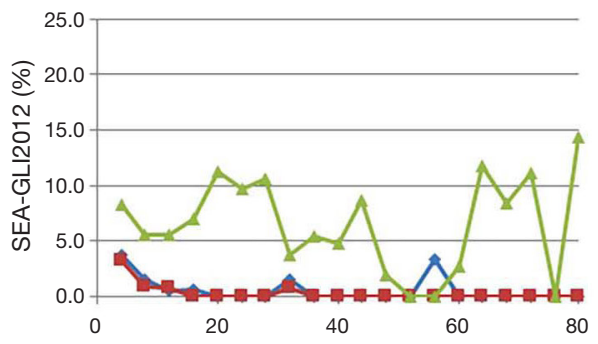

$\rightarrow$ FVC $\%<L L N-$ FEV $_{1} \%<$ LLN - FEV $1 /$ FVC $\%<$ LLN
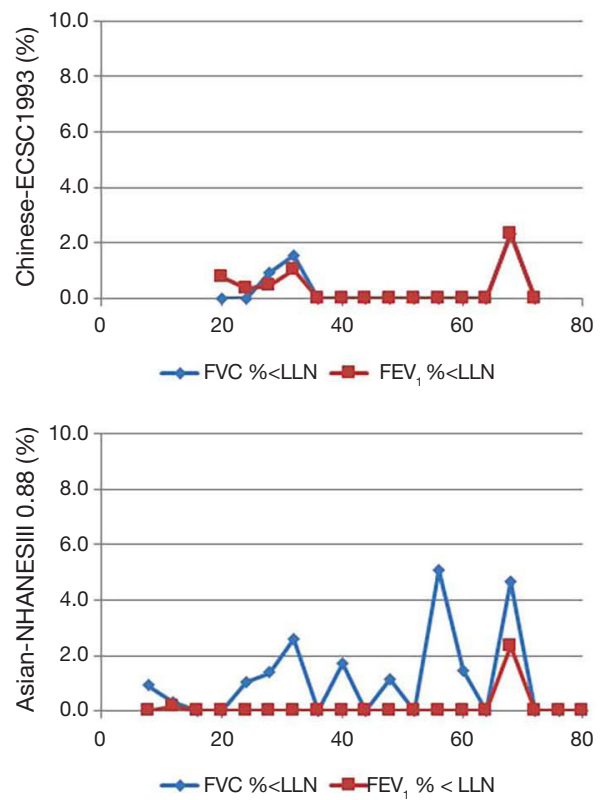

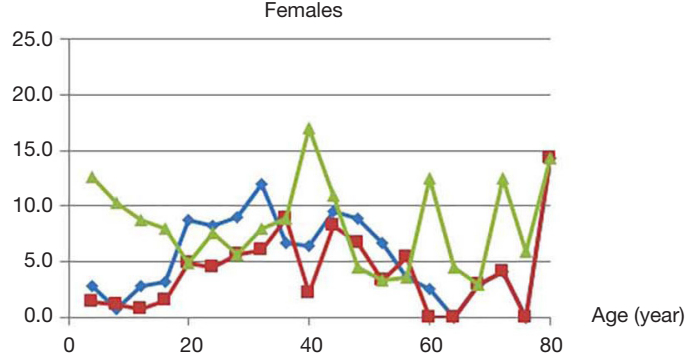

$\leadsto \mathrm{FVC} \%<\mathrm{LLN}-\mathrm{FEV}_{1} \%<\mathrm{LLN} \longrightarrow \mathrm{FEV}_{1} / \mathrm{FVC} \%<\mathrm{LLN}$

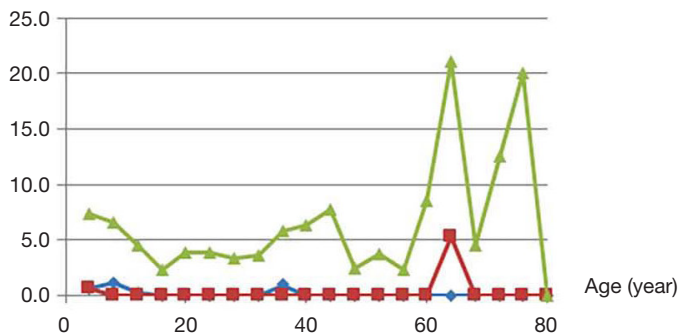

$\rightarrow$ FVC $\%<$ LLN-E-FEV $\%<$ LLN $\rightarrow-\mathrm{FEV}_{1} / \mathrm{FVC} \%<\mathrm{LLN}$

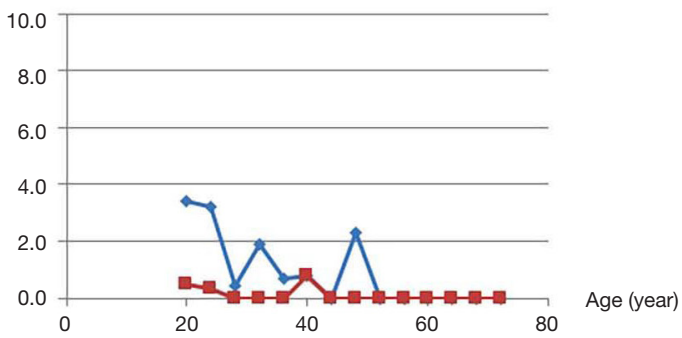

$\rightarrow \mathrm{FVC} \%<\mathrm{LLN} \rightarrow \mathrm{FEV}_{1} \%<\mathrm{LLN}$

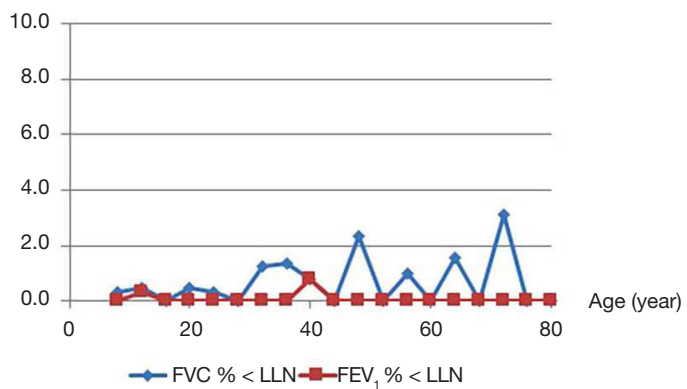

Figure 7 The percentage of the measured values below lower limits of normal (\%<LLNs) across the whole age in FVC (blue line), FEV (red line) and $\mathrm{FEV}_{1} / \mathrm{FVC}$ (green line). The intervals of age are 5 years. SEA-GLI2012 and NEA-GLI2012: multi-ethnic Global Lung Initiative 2012 equations for Southeast and Northeast Asian; Chinese-ECSC1993: European Committee of Steel and Coal equations adjusted for Chinese; Asian-NHANESIII 0.88: the third National Health and Nutrition Examination Surveys equations adjusted with 0.88 times for Asian-American. LLNs, lower limits of normal; FVC, forced vital capacity; $\mathrm{FEV}_{1}$, forced expiratory volume in 1 second. 
Table 2 Validation of SEA-GLI2012, NEA-GLI2012, Chinese-ECSC1993 and Asian-NHANESIII 0.88

\begin{tabular}{|c|c|c|c|c|c|c|c|c|c|c|c|c|c|}
\hline Reference & Age & \multicolumn{4}{|c|}{ FVC (L) } & \multicolumn{4}{|c|}{$\mathrm{FEV}_{1}(\mathrm{~L})$} & \multicolumn{4}{|c|}{$\mathrm{FEV}_{1} / \mathrm{FVC}$} \\
\hline \multicolumn{14}{|l|}{ Males } \\
\hline The present study & $4-80$ & -0.01 & -0.3 & $0.00 \pm 1.00$ & 5.1 & -0.00 & -0.3 & $0.00 \pm 1.00$ & 4.8 & 0.00 & 0.3 & $-0.00 \pm 1.00$ & 4.6 \\
\hline SEA-GLI2012 & $4-80$ & -0.24 & -7.2 & $0.64 \pm 1.04$ & 1.0 & -0.21 & -7.2 & $0.66 \pm 1.00$ & 0.6 & -0.00 & -0.0 & $0.07 \pm 1.05$ & 5.6 \\
\hline Chinese-ECSC1993 & $18-70$ & -0.31 & -6.8 & $0.54 \pm 0.88$ & 0.4 & -0.23 & -6.0 & $0.47 \pm 0.84$ & 0.4 & NA & $\mathrm{NA}$ & NA & NA \\
\hline Asian-NHANESIII 0.88 & $8-80$ & -0.29 & -7.6 & $0.67 \pm 1.00$ & 0.9 & -0.33 & -10.2 & $0.71 \pm 0.80$ & 0.1 & NA & NA & NA & NA \\
\hline \multicolumn{14}{|l|}{ Females } \\
\hline The present study & $4-80$ & -0.01 & -0.4 & $0.00 \pm 1.00$ & 4.6 & -0.00 & -0.3 & $0.00 \pm 1.00$ & 4.6 & 0.00 & 0.3 & $0.00 \pm 1.00$ & 5.2 \\
\hline Chinese-ECSC1993 & $18-70$ & -0.13 & -3.9 & $0.30 \pm 0.97$ & 1.4 & -0.24 & -8.8 & $0.69 \pm 0.90$ & 0.2 & NA & NA & NA & NA \\
\hline Asian-NHANESIII 0.88 & $8-80$ & -0.22 & -7.3 & $0.62 \pm 0.98$ & 0.5 & -0.24 & -9.1 & $0.65 \pm 0.82$ & 0.1 & NA & $\mathrm{NA}$ & NA & NA \\
\hline
\end{tabular}

The predicted values were calculated with age and height from the present sample data. FVC, forced vital capacity; FEV $_{1}$, forced expiratory volume in 1 second; SEA-GLI2012 and NEA-GLI2012, multi-ethnic Global Lung Initiative 2012 equations for Southeast and Northeast Asian; Chinese-ECSC1993, European Committee of Steel and Coal equations adjusted for Chinese; Asian-NHANESIII 0.88, the third National Health and Nutrition Examination Surveys equations adjusted with 0.88 times for Asian-American; $\triangle \%$, percentage differences compared with measured values; LLN\%, percentage of individuals whose measure value below lower limit of normal; NA, not available.

Table 3 Comparisons with other previous reference values derived from Chinese adults

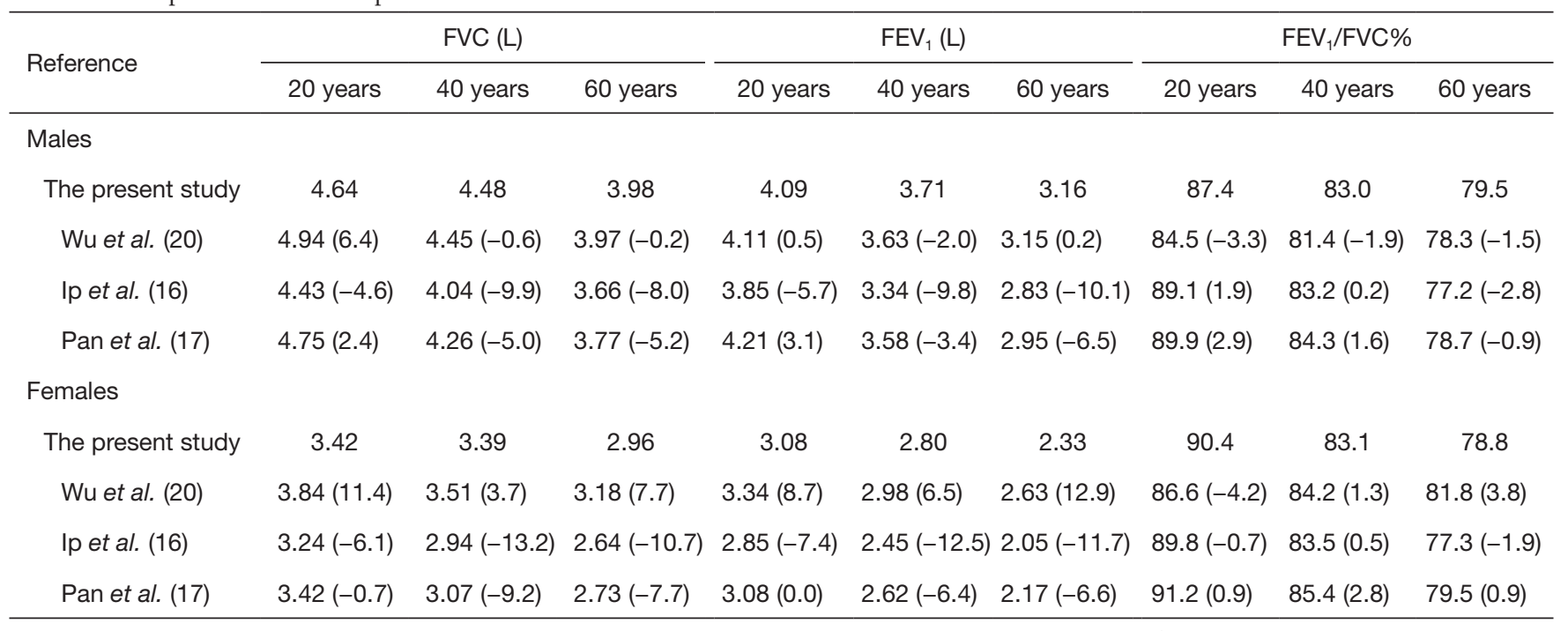

Data are presented as predicted values in male adults with $170 \mathrm{~cm}$ in height and $65 \mathrm{~kg}$ in weight, while in female adults with $160 \mathrm{~cm}$ in height and $55 \mathrm{~kg}$ in weight (\% difference compared with the present study). FVC, forced vital capacity; FEV $_{1}$, forced expiratory volume in 1 second. 
Table 4 Comparisons with previous reference equations derived from Chinese in children

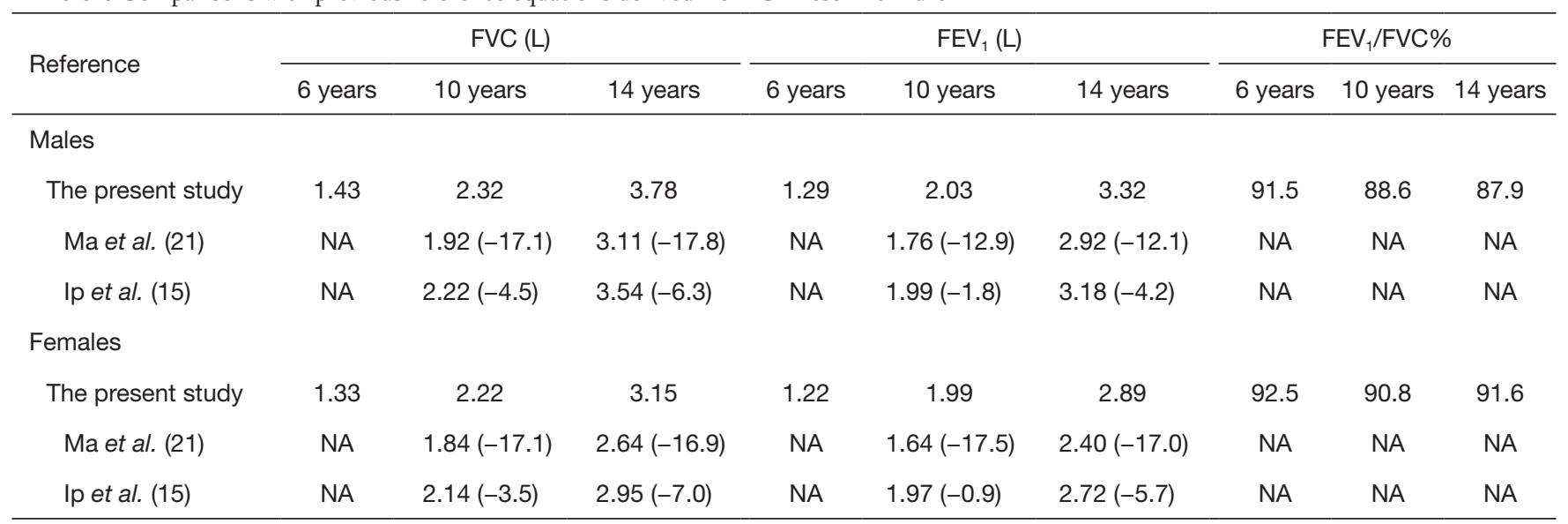

Data are presented as predicted values in children (\% difference compared with the present study). Because of the growth in children, the mean height and weight for age were obtained in the present study. The data of height and weight were $117 \mathrm{~cm}$ and $21 \mathrm{~kg}, 140 \mathrm{~cm}$ and $34 \mathrm{~kg}, 164 \mathrm{~cm}$ and $54 \mathrm{~kg}$, at 6,10 and 14 years old of male children, respectively. The data of height and weight were $117 \mathrm{~cm}$ and $20 \mathrm{~kg}$, $141 \mathrm{~cm}$ and $32 \mathrm{~kg}, 158 \mathrm{~cm}$ and $48 \mathrm{~kg}$, at 6,10 and 14 years old of female children, respectively. FVC, forced vital capacity; FEV , forced $^{2}$ expiratory volume in 1 second; NA, not available.

Wang, Rui Jin Hospital of Shanghai Jiao Tong University School of Medicine, Shanghai 200025, China; Jianzhang Yuan, Guangzhou Red Cross Hospital, Guangzhou 510220, China; Xiaoju Liu, The First Hospital of Lanzhou University, Lanzhou 730000, China.

Funding: This work was supported by Chronic respiratory diseases funding, Chinese Medical Association research projects, National Key Technology R\&D Program (2016YFC1304603, 2015BAI12B10).

\section{Footnote}

Conflicts of Interest: The authors have no conflicts of interest to declare.

\section{References}

1. Global Initiative for Chronic Obstructive Lung Disease (GOLD). Global strategy for the diagnosis, management and prevention of chronic obstructive pulmonary disease, 2017. Available online: http://www.goldcopd.org

2. Global Initiative for Asthma (GINA). Global strategy for asthma management and prevention, 2017. Available online: http://www.ginasthma.org

3. Quanjer PH, Stanojevic S, Cole TJ, et al. Multi-ethnic reference values for spirometry for the 3-95-yr age range: the global lung function 2012 equations. Eur Respir J 2012;40:1324-43.
4. Overseas Chinese Affairs Office of The State Council. The population of overseas Chinese, 2011. Available online: http://www.gqb.gov.cn//news/2011/1201/24945.shtml

5. National Bureau of Statistics of China. The sixth national census, 2011. Available online: http://www.stats.gov.cn/tjsj/ zxfb/201104/t20110428_12705.html

6. National Health and Family Planning Commission of the People's Republic of China. The 2013 China health statistics. Available online: http://www.nhfpc.gov.cn/ mohwsbwstjxxzx/s7967/201404/f3306223b40e4f18a43cb6 8797942d2d.shtml

7. Zheng JP, Kang J, Huang SG, et al. Effect of carbocisteine on acute exacerbation of chronic obstructive pulmonary disease (PEACE Study): a randomised placebo-controlled study. Lancet 2008;371:2013-8.

8. A 24-week Study to Evaluate the Efficacy and Safety of GSK573719/GW642444 125/25 mcg and 62.5/25mcg Inhalation Powder Compared With Placebo in Subjects With COPD. Available online: http://clinicaltrials.gov/ ct2/show/NCT01636713?term=01636713\&rank=1

9. Quanjer PH, Tammeling GJ, Cotes JE, et al. Lung volumes and forced ventilatory flows. Report Working Party Standardization of Lung Function Tests, European Community for Steel and Coal. Official Statement of the European Respiratory Society. Eur Respir J Suppl 1993;16:5-40.

10. Zheng J, Zhong N. Normative values of pulmonary function testing in Chinese adults. Chin Med J (Engl) 
2002;115:50-4.

11. Hankinson JL, Odencrantz JR, Fedan KB. Spirometric reference values from a sample of the general U.S. population. Am J Respir Crit Care Med 1999;159:179-87.

12. Hankinson JL, Kawut SM, Shahar E, et al. Performance of American Thoracic Society-recommended spirometry reference values in a multiethnic sample of adults: the multi-ethnic study of atherosclerosis (MESA) lung study. Chest 2010;137:138-45.

13. Zhang QL, Zheng JP, Yuan BT, Het al. Feasibility and predicted equations of spirometry in Shenzhen preschool children. Zhonghua Er Ke Za Zhi 2005;43:843-8.

14. Leung TF, Liu TC, Mak KK, et al. Reference standards for forced expiratory indices in Chinese preschool children. Pediatr Pulmonol 2013;48:1119-26.

15. Ip MS, Karlberg EM, Karlberg JP, et al. Lung function reference values in Chinese children and adolescents in Hong Kong. I. Spirometric values and comparison with other populations. Am J Respir Crit Care Med 2000;162:424-9.

16. Ip MS, Ko FW, Lau AC, et al. Updated spirometric reference values for adult Chinese in Hong Kong and implications on clinical utilization. Chest 2006;129:384-92.

17. Pan WH, Chen JY, Haung SL, et al. Reference spirometric values in healthy Chinese never smokers in two townships of Taiwan. Chin J Physiol 1997;40:165-74.

18. Jeng MJ, Chang HL, Tsai MC, et al. Spirometric pulmonary function parameters of healthy Chinese children aged 3-6 years in Taiwan. Pediatr Pulmonol 2009;44:676-82.

19. Tsai MC, Jeng MJ, Chang HL, et al. Spirometric reference equations for healthy children aged 6 to 11 years in Taiwan. J Chin Med Assoc 2010;73:21-8.

20. Wu Y, Zhang Z, Gang B, et al. Predictive equations for lung function based on a large occupational population in North China. J Occup Health 2009;51:471-7.

21. Ma YN, Wang J, Dong GH, et al. Predictive equations using regression analysis of pulmonary function for healthy children in Northeast China. PLoS One 2013;8:e63875.

22. Jiang M, Gao Y, Zhong NS, et al. Spirometric reference

Cite this article as: Jian W, Gao Y, Hao C, Wang N, Ai T, Liu C, Xu Y, Kang J, Yang L, Shen H, Guan W, Jiang M, Zhong $\mathrm{N}$, Zheng J. Reference values for spirometry in Chinese aged 4-80 years. J Thorac Dis 2017;9(11):4538-4549. doi: 10.21037/ jtd.2017.10.110 values for healthy Han children aged 5-15 years in Guangzhou, southern China. Pediatr Pulmonol 2015;50:1009-16.

23. Zhong N, Wang C, Yao W, et al. Prevalence of chronic obstructive pulmonary disease in China: a large, population-based survey. Am J Respir Crit Care Med 2007;176:753-60.

24. Li X, Zhou Y, Chen S, et al. Early intervention with tiotropium in Chinese patients with GOLD stages I-II chronic obstructive pulmonary disease (Tie-COPD): study protocol for a multicentre, double-blinded, randomised, controlled trial. BMJ Open 2014;4:e003991.

25. Zheng J. A nationwide questionnaire survey on clinical application of pulmonary function testing in China. Zhonghua Jie He He Hu Xi Za Zhi 2002;25:69-73.

26. Miller MR, Hankinson J, Brusasco V, et al. Standardisation of spirometry. Eur Respir J 2005;26:319-38.

27. Pellegrino R, Viegi G, Brusasco V, et al. Interpretative strategies for lung function tests. Eur Respir J 2005;26:948-68.

28. Quanjer PH, Stanojevic S, Stocks J, et al. GAMLSS in action, 2012. Available online: http://www.ers-education. org/Media/Media.aspx?idMedia=266692

29. Cole TJ, Stanojevic S, Stocks J, et al. Age- and size-related reference ranges: a case study of spirometry through childhood and adulthood. Stat Med 2009;28:880-98.

30. Hall GL, Thompson BR, Stanojevic S, et al. The Global Lung Initiative 2012 reference values reflect contemporary Australasian spirometry. Respirology 2012;17:1150-1.

31. Tian J, Zhou Y, Cui J, et al. Peak expiratory flow as a screening tool to detect airflow obstruction in a primary health care setting. Int J Tuberc Lung Dis 2012;16:674-80.

32. Harik-Khan RI, Fleg JL, Muller DC, et al. The effect of anthropometric and socioeconomic factors on the racial difference in lung function. Am J Respir Crit Care Med 2001;164:1647-54.

33. Maio S, Sherrill DL, MacNee W, et al. The European Respiratory Society spirometry tent: a unique form of screening for airway obstruction. Eur Respir J 2012;39:1458-67. 



Table S3 Reference equations for males and female

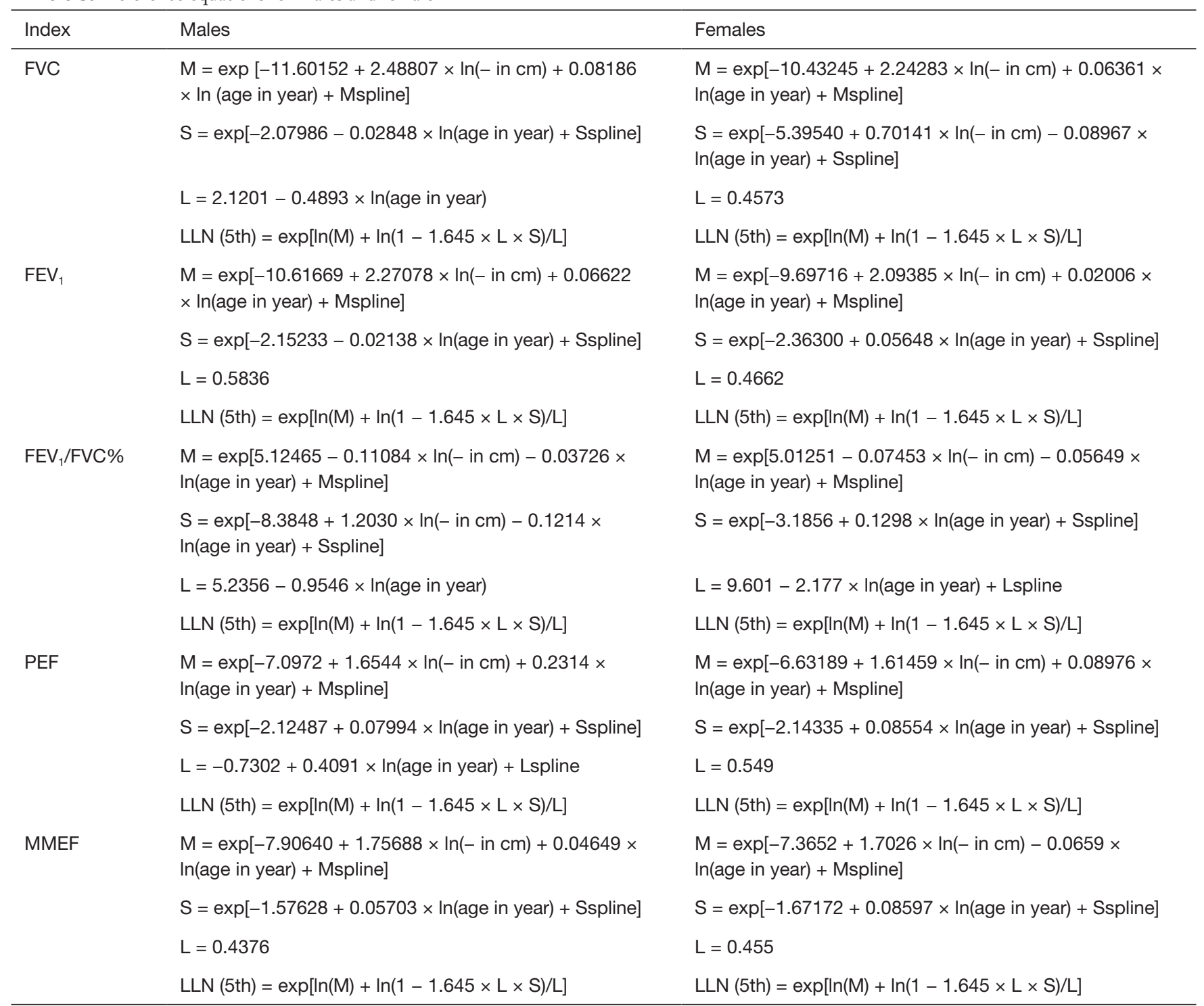

Spline is an age-specific contribution from the spline function. FVC, forced vital capacity; $F_{E V}$, forced expiratory volume in 1 second; PEF, peak expiratory flow; MMEF, maximal midexpiratory flow; LLN, lower limit of normal; M, indicates the predicted value; S, indicates scatter, the coefficient of variation; L, indicates location, the skewness. 